\title{
Données ouvertes et redéfinition de la culture de l'information dans les organisations
}

Vers une culture de la donnée

Open Data and redefinition of information culture within organizations: Toward a culture of data

\section{Anne Lehmans}

\section{CpenEdition} Journals

Édition électronique

URL : http://journals.openedition.org/communicationorganisation/5495

DOI : 10.4000/communicationorganisation.5495

ISBN : 979-10-300-0146-4

ISSN : 1775-3546

\section{Éditeur}

Presses universitaires de Bordeaux

\section{Édition imprimée}

Date de publication : 1 juin 2017

Pagination : 15-26

ISBN : 979-10-300-0162-4

ISSN : $1168-5549$

\section{Référence électronique}

Anne Lehmans, «Données ouvertes et redéfinition de la culture de l'information dans les organisations », Communication et organisation [En ligne], 51 | 2017, mis en ligne le 01 juin 2020, consulté le 01 janvier 2021. URL : http://journals.openedition.org/communicationorganisation/5495 ; DOI : https://doi.org/10.4000/communicationorganisation.5495 


\title{
Données ouvertes et redéfinition de la culture de l'information dans les organisations: vers une culture de la donnée
}

\author{
Anne Lehmans ${ }^{1}$
}

Les organisations et les citoyens voient émerger la centralité et de l'abondance de données - ce que l'on désigne communément par le big data - dans des domaines où apparaissent des acteurs et des enjeux nouveaux. L'horizon se profile d'une économie globale dominée par les grandes entreprises du numérique (les GAFAM) d'un côté, d'une économie circulaire basée sur la création, le partage et l'innovation, ancrée dans une dimension territoriale (l'open data), de l'autre. De nouveaux métiers, les data scientists, se développent autour de la gestion et de l'exploitation des données dans les entreprises et les organisations. L'usage innovant des données, dans le monde du big data, apparaît finalement comme coûteux et complexe, car il suppose l'intervention de spécialistes, outre l'accès limité à des bases de données payantes. En revanche, les données ouvertes (open data) désignent les données primaires collectées par les organismes publics, privés ou les citoyens, accessibles, dont la réutilisation est possible pour tous et précisée par une licence ouverte. Elles sont diffusées dans des formats numériques accessibles et plutôt sur des plateformes nationales ou locales. L'ouverture des données publiques confirme le mouvement démarré en France en 1978 par la loi créant la commission d'accès aux documents administratifs. Le mouvement d'ouverture des données s'est accéléré depuis la création d'un grand portail data.gouv.fr en 2011 dans sa première version et de la mission Etalab, les lois Valter (sur la gratuité des informations publiques) en 2015 et Lemaire (loi pour une République numérique) en 2016. Mais cette accélération des politiques publiques ne va pas nécessairement de pair avec leur mise en oeuvre dans les organisations, parce que l'ouverture des données représente un changement profond, du point de vue du management de l'information notamment. Elle a pour objectif affiché la transparence, la participation citoyenne et la modernisation de l'action

1 Anne Lehmans est maître de conférences en sciences de l'information et de la communication à l'université de Bordeaux, chercheuse à l'IMS-RUDII. Elle travaille sur les cultures de l'information dans les contextes éducatifs et professionnels ; anne.lehmans@u-bordeaux.fr 
publique (E1 Hachani 2015 : 5). Mais le discours de la transparence relève en grande partie d'une utopie (Gallot, Verlaet 2016), celui de la participation citoyenne d'une complexité qui requiert la prise en compte des dimensions pragmatiques de la politique (Zask 2014), et celui de l'innovation, liée à la modernisation de l'action publique, d'une forme d'instrumentalisation de la gouvernance (Lascoumes, Simard 2011) à travers un récit performatif. On peut alors se demander à quelles conditions les organisations peuvent véritablement coproduire et se saisir des données ouvertes.

Un projet Data-cultures vise à intégrer les données ouvertes à des usages didactiques et pédagogiques, dans une perspective de construction de la culture de l'information. Dans le cadre de ce projet, la description et l'analyse des processus de production et de diffusion des données ouvertes (la gouvernance des données) ont permis de situer ces usages dans le cycle de vie de la donnée. Or, il est très vite apparu que dans les organisations, la mise en place plus ou moins spontanée de procédures d'ouverture des données soulevait des problèmes qui relevaient, plus que de questions techniques et juridiques, de questions culturelles liées à des savoirs, des pratiques, des valeurs et des représentations partagés (Liquète 2014). Simon Chignard, expert et militant de l'ouverture des données, parle d'une "bataille culturelle " à mener pour trois acteurs : les producteurs de données qui les diffusent, les utilisateurs de la donnée primaire qui créent des applications ou des services par exemple, et les utilisateurs finaux, les usagers. On peut ajouter un quatrième acteur qui a émergé sur la scène de la donnée ouverte, dans la brèche culturelle qui s'est révélée, les médiateurs. L'épaisseur culturelle et sociale de la donnée naît ainsi d'une construction dynamique qui relève d'un dialogue entre ces acteurs à l'intérieur et avec l'extérieur des organisations dans un écosystème informationnel où cristallisent la prescription, la gestion et la médiation.

Ce projet de recherche sur la médiation des données ouvertes en éducation a permis de définir les éléments d'une culture de la donnée en construction dans les organisations qui produisent des données ouvertes. Il est nécessaire d'expliquer les fondements méthodologiques d'une analyse des processus de construction et de mobilisation de la culture de la donnée dans les organisations qui diffusent des données. Le terrain concerné est celui de collectivités territoriales en région aquitaine qui ont mis en place une politique d'ouverture des données. Cette culture se définit par rapport à une demande sociale diffuse et dans un cadre organisationnel qui doit être précisé. Elle a fait émerger un marché de la médiation pour sa pérennisation.

\section{Les processus de construction et de mobilisation de la culture de la donnée dans les organisations}

La circulation de l'information dépend des interactions entre les individus dans les organisations, de leurs compétences, des contextes d'exercice et des appareillages techniques. Il existe des écarts conséquents entre les offres 
potentielles de données ouvertes et la réalité de pratiques professionnelles au quotidien, extrêmement fragmentées (Pinède 2009), peu efficaces. Partant de ces constats, le projet Data cultures vise à caractériser la chaîne de production des données ouvertes, en regard de la chaîne d'usages, notamment dans les contextes de formation, et à cartographier l'écosystème des données. Si la question des données ouvertes évolue rapidement dans les politiques, du local à l'international, la réalité des pratiques en France, en comparaison des pratiques canadiennes, par exemple, reste hétérogène. Le Secrétariat général pour la modernisation de l'action publique a remis en 2015 un rapport sur "La gouvernance de la donnée » qui pointe les limitations dans le potentiel des usages des données, liées à la méconnaissance, à l'imperméabilité de la culture administrative par rapport aux dynamiques de coopération et de participation. Ce rapport souligne le fait que «la mise à disposition de données libres et ouvertes, et leur réutilisation, génèrent de la valeur économique et sociale, par le biais de cinq mécanismes : la réduction des coûts de transaction, l'innovation, la réduction des asymétries d'information, la collaboration et les boucles de rétroaction » (p. 21). Il préconise de partir de développements concrets, de cartographier les données disponibles, de faire évoluer les systèmes d'information et de décloisonner les administrations. Pour que ces politiques soient efficaces, notamment au niveau territorial de la région qui est particulièrement concerné, elles doivent rencontrer des besoins, des représentations et des usages, une culture partagée de la donnée. Il s'agit non seulement d'assurer l'accessibilité de méthodes et savoir-faire pour créer et exploiter les données ouvertes, mais aussi de construire activement une démocratie participative dans le contexte émergent des " villes intelligentes » tout en favorisant le développement économique régional, notamment dans le cadre de l'économie sociale et solidaire.

Les politiques d'ouverture des données mises en place ne rencontrent pas encore l'adhésion des organisations pour un passage à l'échelle globale, malgré l'existence de nombreuses expériences locales très positives. Le projet sur la médiation des données pour l'éducation a pour but d'analyser les conditions de construction d'une véritable culture de l'information intégrant la donnée chez les enseignants qui pourraient en faire un usage pédagogique. Il met en relation la question de l'éducation et de la formation aux données avec celle de leur gouvernance dans les organismes publics et privés chargés de les diffuser, qui appelle de nouvelles compétences et connaissances. Il s'intéresse à la façon dont se construit l'offre de jeux de données au sein de l'écosystème informationnel régional, et aux liens à construire avec les professionnels de l'éducation, de la formation et les associations pour former aussi bien les jeunes que tous les acteurs de la société aux données et à leur appropriation. Il s'agit de faire émerger une culture partagée des données, enjeu de leur réappropriation effective par la société civile et dans les entreprises. Mais cette culture prend racine dans les organisations qui produisent les données. 
La question est à considérer dans le cadre large de l'émergence, dans le champ des techniques, des savoirs, et des imaginaires, de la donnée comme élément central des stratégies d'information et de connaissance. Cette émergence correspond à un mouvement d'externalisation de la connaissance vers l'environnement (Cardon 2014, 2015). La pyramide de la donnée à l'information, à la connaissance et à la sagesse (Ackoff 1989), transformée en chaîne de la donnée à la compétence dans le Glossaire de la donnée publique (2016), est une métaphore très claire de la place centrale de la donnée comme élément de base : de l'information en général, des informations personnelles, de la capacité à agir sur le monde en produisant et en utilisant des données de façon éclairée. Dans les organisations, cette valorisation de la donnée, qui ne peut passer que par des procédures expertes, partagées et normalisées, généralisées à un grand nombre de services, est une nouveauté qui exige des formes d'acculturation. Il ne s'agit plus seulement de produire et faire circuler l'information, mais de remonter à la naissance de l'information, la donnée en étant l'élément de base. Le rapport de l'administrateur général des données $(2015,13)$ définit la donnée numérique comme « la description élémentaire de nature numérique, représentée sous forme codée, d'une réalité (chose, évènement, mesure, transaction, etc.) en vue d'être : collectée, enregistrée, traitée, manipulée, transformée, conservée, archivée ». Pour les professionnels, le passage de compétences centrées sur l'information à des compétences de la donnée indispensables pour réaliser l'ensemble des opérations citées, tout en comprenant les enjeux économiques, sociaux, politiques, est un saut culturel, dans la mesure où des représentations, des pratiques et des compétences doivent être construites et partagées.

Le projet de recherche a permis d'enquêter sur le terrain de collectivités locales productrices de données auprès d'une quinzaine d'acteurs (le département de la Gironde, Bordeaux Métropole, le grand Poitiers et le Groupement d'Intérêt Public Aménagement du Territoire et Gestion des Risques en Aquitaine), des médiateurs et des utilisateurs finaux des jeux de données ouvertes. Les objectifs initiaux du projet sont d'identifier:

- les représentations des usages possibles et les usages réels des données,

- les stratégies et les moyens de gouvernance à travers la sélection et la mise à disposition des données,

- les stratégies de communication, de formation, de médiation mises en place.

Léquipe de recherche a mené des observations participatives auprès des producteurs, des médiateurs et des utilisateurs de données, avec des séances de travail sous forme d'ateliers et de focus groupes, afin de comprendre les contraintes et les freins éventuels au développement d'une culture des données, ainsi que les conceptions de cette culture qui circulent dans les organisations. En parallèle des observations qui portent sur des groupes, l'analyse des discours des acteurs de terrain permet de cerner leurs représentations 
ainsi que les objectifs qu'ils se fixent dans le processus d'acculturation aux données ouvertes qui accompagne le cycle de production des données. Des entretiens compréhensifs ont permis de voir comment s'articule la chaîne de production et de diffusion des données dans la sphère publique actuelle, de comparer les représentations d'acteurs entre des secteurs d'activités et des échelles géographiques différents, et de saisir l'évolution de leurs pratiques déclarées sur leurs terrains. La diversité des pratiques peut être l'objet de questionnements méthodologiques sur la formalisation des ressources de formation et de médiation à mettre en œuvre auprès des professionnels. Ces derniers travaillent dans des organisations impliquées dans la publication, la mise à disposition, la valorisation de données ouvertes (producteurs et médiateurs).

\section{Une nécessité sociale et organisationnelle}

La donnée représente un enjeu majeur de culture et s'en préoccuper dans une perspective de formation relève d'une responsabilité politique forte. On choisit ici de parler de culture et pas de compétences ou de connaissances, parce que c'est véritablement un ensemble de représentations, un univers de sens, une capacité à se projeter, un sentiment d'appartenance qui sont en jeu, et pas seulement la capacité à manipuler des outils informatiques. Cette culture se cristallise et se transforme autour des données, comme élément de base de l'information. L'émergence d'une nouvelle problématique professionnelle dans des organisations comme les collectivités territoriales a fait ressortir le fait que les procédures et les routines professionnelles étaient souvent mal adaptées pour répondre à cet enjeu nouveau de l'ouverture des données. Les services organisés en silos fonctionnels, communiquant peu entre eux, se sont vus confrontés à un enjeu commun et transversal, qui les obligeait à revoir, d'une part l'organisation globale des procédures de collecte et de gestion des données, d'autre part les compétences métiers, et par-dessus tout, le partage d'une vision commune de la donnée. Au-delà des compétences techniques, notamment informatiques, mais aussi liées aux méthodes de collecte et de relevé des données, c'est véritablement un ensemble de pratiques informationnelles, de représentations techniques et sociales et de savoirs partagés qui est en jeu, ce qui définit le cœur même d'une culture et de la construction de l'identité professionnelle.

On peut repérer trois grandes caractéristiques récurrentes dans la culture de la donnée en émergence, qui renvoient à un ensemble de valeurs :

- La pluralité

La culture des données se construit en surplomb d'un paysage morcelé de compétences spécialisées diverses qui correspondent aux " métiers » ou aux «thématiques » dans les services. Les organisations fonctionnent avec ces métiers qui constituent l'identité professionnelle des agents, le socle de leurs pratiques, de leurs routines, de leurs représentations, des interactions ou 
des domaines réservés. L'introduction d'une nouvelle problématique comme celle de l'ouverture des données ne peut se faire sans tenir compte de cet existant. Ainsi, pour un responsable de la gestion des données ouvertes dans une collectivité, les changements ne peuvent partir que des pratiques réelles des personnes. Il affirme avoir

[...] essayé de mener ce projet en étant sur place, en travaillant avec les gens, au contact, en m'imprégnant bien de la culture métier et puis en me rendant plus familier en passant deux jours par semaine sur le site. Donc maintenant je connais bien le métier, $[\ldots]$ j’ai inventorié tout un tas de connaissances numériques qui passent totalement sous le radar de la collectivité. Aussi bien DRH que de l'encadrement de proximité. Il n'y a aucun travail dans la collectivité qui est fait de cartographier les compétences.

La connaissance des compétences « métier» existantes est doncindispensable. Les représentations sont également hétérogènes dans les organisations. Ainsi, au sein d'une communauté urbaine, un responsable pointe l'opposition entre une conception de l'ouverture des données basée sur la transparence de la vie publique, et une conception " orientée business ", entre le point de vue des fonctionnaires territoriaux spécialisés dans des services et celui des élus, surplombant mais peu expert, et, dans la culture administrative des fonctionnaires, celle qui est spécialisée dans des fonctions techniques transversales (le service informatique par exemple) ou thématiques, et celle qui est plus politique (le service communication par exemple). La culture de la donnée relève donc d'un processus de négociation.

- La transversalité

Dans la diversité des références professionnelles qui engagent un rapport différent à l'information dans le cadre des missions de chacun, la question des données ouvertes impose une dynamique transversale. Un agent dit appartenir à la direction du système d'information mais " ne pas être issu d'un cursus scientifique technique ». Pour lui, la diversité des cultures professionnelles et des points de vue est un atout, " soit en géomatique, soit en communication, soit sur toutes ces questions de médiation numérique ». Cette diversité permet de créer des ponts entre les différents aspects de gestion de la donnée, qui ne se limitent en aucun cas à la gestion informatique de bases de données : la documentation, l'archivage, la gestion des documents, sont des aspects essentiels soulignés par cet agent, qui vient du monde de l'archivistique et trouve sa place dans un service chargé des open data, en puisant à la fois dans sa culture d'origine, qui le sensibilise à l'importance de la description des données, des procédures, des standards et des classements, et dans une culture informatique acquise sur le tas, qu'il qualifie de " culture du pillage et de la mise à disposition de ressources ». A l'instar de la culture de l'information, le terme « culture de la donnée » n'est pas employé dans les pays anglo-saxons qui préfèrent parler de data literacy, définie comme «le désir et 
la capacité de s'engager de façon constructive dans la société à travers et avec les données $»^{2}$. Cette dernière est centrée sur une conception de la technique comme étant au service des buts et des capacités des hommes, considérée comme un processus relevant d'une démarche active incluant les personnes qui n’ont pas les compétences nécessaires pour manipuler des jeux de données, et sur une définition très large de la donnée comme "item d'information ". Même s'il existe des différences entre la version francophone centrée sur les savoirs et les apprentissages, et la version anglophone centrée sur les activités et les processus, les deux conceptions insistent sur une dimension collective et volontaire, engagée, de l'agir avec et sur la donnée. Ainsi, on trouve parmi les acteurs des données ouvertes dans les collectivités locales des militants du libre avec Open Street Map notamment, auquel ils peuvent consacrer des temps de formations à leurs collègues ou aux usagers.

- La technicité liée aux nécessités de partage

Les politiques d'ouverture des données supposent nécessairement des pratiques collaboratives dans une logique de partage de l'information, tant à l'intérieur de l'organisation entre les services qu'à l'extérieur avec d'autres organisations. Les données géographiques ont été les premières concernées par ces politiques. La directive INSPIRE de la Direction générale de l'environnement de la Commission européenne datant de 2007, «vise à établir en Europe une infrastructure de données géographiques pour assurer l'interopérabilité entre bases de données et faciliter la diffusion, la disponibilité, l'utilisation et la réutilisation de l'information géographique en Europe» (Conseil national de l'information géographique). Dans le cas des données géographiques, c'est donc au niveau politique et à l'échelon européen que des standards ont été imposés afin d'obtenir une information structurée, mise à jour et partageable, qui a permis le développement d'outils comme le Géocatalogue et le Géoportail. L'application de cette directive oblige, dans l'organisation chargée de l'information géographique, les thématiciens et les informaticiens à adopter des normes, des langages, des pratiques communs visant l'interopérabilité des données. Pour une responsable interrogée, il s'agit d'une véritable culture, technique et normative, qui a donné aux services d'information géographique une longueur d'avance sur les collectivités nouvellement arrivées dans l'open data, peu sensibilisées aux nécessités fortes de standardisation et à des outils techniquement complexes, qui appellent de nouvelles compétences. On voit ainsi que la problématique de l'open data a fait émerger une culture nouvelle qui ne porte plus sur des domaines d'information spécialisés mais sur une base nécessairement commune de traitement de la donnée pour offrir son accès, son usabilité, sa pérennisation. La métadonnée apparaît ainsi comme un élément pivot de la culture technique de la donnée, cristallisant des enjeux de compétence informationnelle.

2 Traduction du dictionnaire proposé par Data Pop Alliance, disponible à : http://datapopalliance.org/ resources/key-terms/\#data-literacy 
À ces caractéristiques, on peut ajouter celle de l'engagement autour de valeurs communes que l'on rencontre fréquemment parmi les acteurs les plus mobilisés autour des données ouvertes, qui croise la dimension critique de la culture des données. Finalement, on peut faire le rapprochement entre cette culture de la donnée, centrée sur l'élément de base de l'information et non sur l'information elle-même, mais le situant dans le processus de circulation et de communication de l'information, et la notion de translittératie. Cette dernière repose sur un " triple présupposé anthropologique, techniciste et socioéconomique » (Liquète, Delamotte, Chapron 2012). Quand on considère la culture de la donnée dans les organisations, il ne s'agit pas seulement d'une culture informatique, mais d'une convergence entre la capacité à produire et traiter des données informatiquement, l'appréhension de la donnée dans un écosystème informationnel ouvert qui met en jeu son cycle de vie, et la compréhension des enjeux sociaux, politiques, économiques de la production de données ouvertes. Tous les professionnels interrogés témoignent du fait que lorsque cette culture est inexistante ou faible, parce que la décision de partager les données est récente, ou prise par un service qui n'est pas au cœur du cycle de production des données (la communication par exemple), la mobilisation des acteurs est très faible et les procédures mises en place peu efficaces pour atteindre cette nécessaire convergence.

\section{Les conditions de la diffusion d'une culture de la donnée dans les organisations}

La culture de la donnée est centrée sur un objet technique mais en appelle une approche non spécialisée et partageable. Il s'agit donc de comprendre à quoi sert une donnée et comment chacun peut agir sur ou faire avec les données, sans se limiter aux manipulations techniques des données. Lobjectif de la culture de la donnée est de s'assurer que tous les membres d'une organisation savent ce que c'est et à quoi ça sert, mais pas nécessairement comment on s'en sert. Cette approche de la technique comme objet complexe et social est proche de celle des « libristes », pour qui le libre n'est pas une affaire d'informaticiens uniquement mais une affaire commune, autour de laquelle les connaissances à construire sont essentiellement sociales et politiques : chacun doit savoir à quoi sert l'informatique et comment agir pour que la technique produise ce que chacun et tous considèrent comme souhaitable, sans être capables de la manipuler (Data Pop Alliance 2015).

Louverture des données, dans les principes, relève d'un projet ou d'un discours de partage, de transparence, de diffusion démocratique et égalitaire des bases de la connaissance. Or, si l'on considère la réalité des usages des données ouvertes au niveau d'une région par exemple, on constate que les réutilisations réelles et projetées par les diffuseurs concernent essentiellement un marché. À l'intérieur même des organisations comme les collectivités territoriales, la culture de la donnée se construit très diversement, en 
fonction de lorganisation des services, des prises de position des acteurs et de leur capacité à se faire entendre en bousculant les équilibres mis en place notamment autour des systèmes d'information. Les élus sont souvent peu mobilisés parce que la question des données ouvertes est technique et complexe. Des acteurs nouveaux apparaissent autour des enjeux de médiation, qui installent la culture de la donnée au cœur de leur action et en font un levier pour la création d'un " espace public numérique ». Dans les organisations, la préoccupation de construction d'une culture de la donnée dépend, dans son existence et dans son intensité, des conditions de mise en place d'une politique d'open data. L'impulsion de l'ouverture peut venir de services de communication, des services informatiques, des services techniques, des directions générales ou, beaucoup plus rarement, des élus. Les services qui assurent des missions techniques dans le cycle de vie de la donnée ne partagent pas nécessairement des représentations positives de l'open data. Ils y voient des contraintes nouvelles, des risques de bouleversement des procédures de travail en place, de remise en cause de leur mission par la publicité faite aux données statistiques, par les nécessités de standardisation ou de description dans les métadonnées, mais rarement des objectifs valorisants. Quand l'histoire de l'ouverture des données se fait sur ce modèle, la construction d'une culture de la donnée est longue et vient plutôt de l'émergence d'une demande sociale ou politique. Quand l'impulsion vient de l'intérieur, par exemple de la direction des services informatiques, le cheminement est différent et la construction d'une culture partagée, parfois sur fond de compétition entre les services, plus rapide. Pour certaines organisations comme le groupement d'intérêt public, l'existence d'une culture de la donnée va de soi, non à partir d'une action de formation ou de communication, mais simplement à partir des besoins qui s'imposent à travers leur mission.

Ainsi, la Fondation Internet Nouvelle Génération (FING), par exemple, a construit une grande partie de son activité depuis 2009 autour de la question de la culture de la donnée, en partant du constat de l'importance centrale de la donnée pour les entreprises et de la nécessité de former les professionnels de façon transversale et pas seulement technique. Le programme Infolab de la Fing est donc orienté vers des formes de médiation qui s'appuient sur l'échange, la discussion, la réflexion collective entre des professionnels de secteurs et de compétences très différents (Népote 2016), et non sur des formations spécialisées pour data scientists par exemple ou centrées sur l'expertise informatique. Ce travail de réflexion et d'animation de la FING, inspiré de la démarche qualité, est accompagné par des chercheurs en sciences de l'information et de la communication et un ensemble d'experts. Il s'appuie sur une vision de la culture des données basée sur le "potentiel collaboratif » (Noucher 2011) et le partage. Il a contribué à la définition de la culture de la donnée en opposition à la culture de l'information, à partir de la définition des deux termes : la donnée, " élément défini et isolable qui va pouvoir être 
manipulé, traité et analysé en fonction d'un objectif ou d'un cadre d'analyse » (Labelle, Gilliard 2017), est la base de l'information, qui est elle-même « une donnée interprétée par une personne ». Les ateliers InfoLab consistent à faire émerger une représentation commune de la donnée dans les organisations, dans un processus d'acculturation aux données en trois phases, l'amorçage, le déploiement et la généralisation. On voit donc qu'un marché de la médiation s'installe autour de la culture de la donnée dans les organisations.

La culture de la donnée est composée de savoirs, de valeurs et de compétences statistiques, informatiques, médiatiques, et informationnelles qui donnent les moyens aux professionnels de comprendre le sens de leur activité de collecte et de mise en circulation des données, de la situer dans son contexte social d'usage, et de coordonner les gestes et les procédures professionnels. Cette culture est nécessairement propre à chaque organisation qui construit ses pratiques. Elle parvient à un point de maturité quand les professionnels partagent une vision commune de la donnée, de ses usages, de sa circulation, et des techniques et médias qui permettent de la traiter. Elle n'est pas affaire de spécialiste mais plutôt de représentation partagée d'un écosystème.

\section{BIBLIOGRAPHIE}

ACKOFF RUSSELL L., 1989, «From data to wisdom», Journal of applied systems analysis, vol. 16, p. 3-9.

CARDON Dominique, 2015, A quoi rêvent les algorithmes, Paris, La République des idées.

CARDON Dominique, 2014, «Apprendre/désapprendre: sur la ligne de crête des apprentissages numériques», InternetActu.net, Disponible à: http://www.internetactu. net/2014/01/10/apprendredesapprendre-sur-la-ligne-de-crete-des-apprentissagesnumeriques/.

CHIGNARD Simon, 2012, L'open data, comprendre l'ouverture des données publiques, Paris, Editions Fyp.

DATA-POP ALLIANCE (Harvard Humanitarian Initiative, MIT Media Lab and Overseas Development Institute) and Internews, 2015, «Beyond Data Literacy: Reinventing Community Engagement and Empowerment in the Age of Data.», DataPop Alliance White Paper Series, Septembre, disponible à : http://datapopalliance.org/wpcontent/uploads/2015/10/BeyondDataLiteracy_DataPopAlliance_Sept30.pdf

DEBOS Franck, 2017, « Open data et culture de la donnée: le cas OpeNRJ», Revue française des sciences de l'information et de la communication, 10, disponible à: http://rfsic. revues.org/2639

EL HACHANI Mabrouka, 2015, "Open data, collectivités et usagers: une dynamique en question », in Paquienséguy F., dir., Open data. Accès, territoires, citoyenneté: des problématiques info-communicationnelles, Paris, Éditions des archives contemporaines, p. 1-23. 
FABRE Isabelle, GARDIÈS Cécile, 2012, «Définition et enjeux de la médiation numérique documentaire », in Gallup, X. (Dir.), Développer la médiation documentaire numérique, Villeurbanne, Presses de l'enssib, disponible à: http://mediationdoc.enssib. fr/lire-en-ligne/sommaire/i-le-perimetre-de-la-mediation-numerique-documentaire/ definition-et-enjeux-de-la-mediation-numerique-docu.

FING, 2017, «Vers une organisation data driven : mettre la culture des données au cœur de l'organisation », disponible à : http://infolabs.io/cd16.

FING, 2016, "Campagne Infolab. Développer une "culture de la donnée” au service des entreprises et des acteurs du territoire ", disponible à : http://reseau.fing.org/groups/ profile/124895/campagne-infolab

FRANCE, Secrétariat général pour la modernisation de l'action publique, 2015, Rapport de l'administrateur général des données. Les données au service de la transformation de l'action publique, disponible à : http://www.modernisation.gouv.fr/sites/default/files/ fichiers-attaches/rapport_agd_decembre2015.pdf

GALLOT Sidonie, Verlaet Lise, 2016, « La transparence: l'utopie du numérique?», Communication E Organisation, 1-49, p. 203-217.

LABELLE Sarah, GILLIARD Armelle, 2017, in ENS Cachan, MOOC Éducation aux médias et à l'information à l'ère du numérique (eFAN), France Université Numérique. http://reseau.fing.org/groups/profile/124895/campagne-infolab

LASCOUMES Pierre, SIMARD Louis, 2011, «L'action publique au prisme de ses instruments. Introduction", Revue française de science politique, 1-61, p.5-22.

LIQUÈTE Vincent, 2014, Cultures de l'information, Paris, CNRS Éditions.

LIQUĖTE Vincent, MAUREL Dominique, 2013, «Enquête canado-française GRICODD: Pratiques informationnelles, communicationnelles et documentaires durables». 5e Congrès des milieux documentaires du Québec, Montréal (Québec), 27 novembre 2013.

LIQUÈTE Vincent, DELAMOTTE Eric, CHAPRON Françoise, 2012, « Introduction », Études de communication, 38, p. 9-22.

NÉPOTE Charles, 2016, «Organisations : Pourquoi vous devez vous préoccuper de la culture "data” de vos collaborateurs ", L'Usine Digitale, septembre, disponible à : http:// www.usine-digitale.fr/article/organisations-pourquoi-vous-devez-vous-preoccuper-dela-culture-data-de-vos-collaborateurs. N435337

NOUCHER Matthieu, 2011, «De l'open data à l'innovation distribuée : exploration du potentiel collaboratif de la réutilisation des données publiques ", Colloque annuel de l'AGMQ, May 2011, Québec, Canada.

NOYER, Jean-Max, CARMES Maryse, 2012, «Le mouvement "Open Data” dans la grande transformation des intelligences collectives et face à la question des écritures, du web sémantique et des ontologies. », disponible à : https://archivesic.ccsd.cnrs.fr/ sic_00759618

OPEN DATA FRANCE, La Reine Merlin,2016, Guide Open Data pour les communes/ Glossaire de la donnée publique, disponible à : http://www.opendatafrance.net/wp-content/ uploads/2016/06/guideOD_communes_glossaire_juin2016_Web.pdf 
ZASK Joëlle, 2013, " Pragmatisme et participation », in Casillo I. et al. (dir.), Dictionnaire critique et interdisciplinaire de la participation, Paris, GIS Démocratie et Participation, disponible à : http://www.dicopart.fr/fr/dico/pragmatisme-et-participation

Résumé : Louverture des données dans le mouvement des open data permet de redéfinir la culture de l'information dans les organisations concernées autour d'acteurs, d'enjeux politiques et d'écosystèmes informationnels émergents.

Mots-clés : open data, culture de l'information, compétence informationnelle, écosystème informationnel.

Abstract: The open data movement redefines the culture of information with data literacy in organizations, focusing on emerging actors and political and information issues.

Keywords: open data, data literacy, information culture, information ecosystem. 